\title{
Family experiences about women with breast cancer: A content analysis study
}

\author{
Zeinab Younes Barani $^{1,(D)}$, Mozhgan Rahnama ${ }^{1,} \bowtie,\left(\mathbb{D}\right.$, Mahin Naderifar1, $^{1}$, Elahe Assadi Bidmeshki ${ }^{1,(D)}$, and Hossein Rashki- \\ Ghalenow $^{2, \text { iD }}$
}

${ }^{1}$ Department of Nursing, Nursing and Midwifery School, Zabol University of Medical Sciences, Zabol, Iran

${ }^{2}$ Nursing and Midwifery School, Zahedan University of Medical Sciences, Zahedan, Iran

Purpose: The family members of cancer patients experience many issues after the diagnosis and treatment of their patients. Therefore, this study aimed to explain the experience of family members of women with breast cancer. Methods: This qualitative study is a conventional content analysis approach. Purposeful sampling was performed using seven family members of women with breast cancer. The data collection method was a semistructured interview. The content analysis method was used to identify the categories. Results: Three categories were extracted from the data as mental care while emotional conflict, compassionate efforts in painful moments of care, the development of the ability to cope with care-treatment challenges. Conclusion: Caring for breast cancer patients was a painful and conflicting duration for family members. However, they experienced the growth of some capabilities in themselves. The extracted essence of experiences was "trying to save the patient." Nurses can apply familycentered strategies to create a suitable situation for clients who engage with breast cancer.

Keywords: Breast, Caregiver, Family, Neoplasms

Corresponding: rahnama2030@gmail.com; Received: November 10, 2020; Accepted: November 25, 2020; Published: January 1, 2021; This article is available on https://www.jnexp.ir

\section{Introduction}

Breast cancer and its treatments can make a difference in the lives of patients and their families [1] because they experience many side effects and issues during treatments [2]. Since the family is the first source of care and support for a member with the disease, the family attitude towards the disease is essential [3]. Family members are most involved in caring for the patient, helping her to adapt and manage the condition [4]. However, increasing pressure on caregivers will have several consequences, such as inadequate patient care, patient abandonment, family isolation, despair, and restraint of family relationships [5]. On the other hand, as caregivers spend most of their time with the patient, they often forget about their needs. Cancer affects the whole family [4]. They may experience economic, social, physical, psychological, and spiritual problems [6,7]. Therefore, supporting caregivers of cancer patients during the progression of the disease is very important, because the physical health of the caregivers and their information and knowledge have a profound effect on providing care for the patients [8]. Overall, given the profound effects of breast cancer on the patient and family, it is crucial to be aware of their experiences of this phenomenon. Understanding the experiences is can help nurses to implement family-centered strategies for breast cancer patients [3]. As the quantitative research methods do not have enough capacity and the ability to address such issues, it is necessary to conduct a more in-depth study using a qualitative approach to investigate the phenomenon by gaining the participants' experiences. This study can be a guide for implementing therapeutic care strategies for the treatment team. Therefore, this study aimed to explain the family experiences of women with breast cancer using content analysis. 


\section{Methods}

\section{Ethical approval}

The ethical consideration of the study was approved by the ethical research committee of the Zabol University of Medical Sciences, Iran (IR.ZBMU.REC.1397.149). Ethical principles were observed in the research, including obtaining a letter of introduction, obtaining informed consent from the participants to record the interview, maintaining the confidentiality and their authority to leave the study.

\section{Study design/Setting}

The current study was a qualitative conventional content analysis, which was conducted in the chemotherapy clinic of Imam Khomeini Hospital in Zabol, Iran, in 2017.

\section{Participants}

The study samples were seven individuals of family caregivers of women with breast cancer. Participants were selected through the purposive sampling method. The participants' characteristics are presented in Table 1.

\begin{tabular}{lllllll}
\multicolumn{2}{l}{ Table 1. Profile of family caregivers } \\
\hline Participant & Age & Marriage status & Job & Education level & Relationship & Care duration \\
\hline $\mathbf{1}$ & 37 & Married & Housewife & Diploma & Daughter & 7 months \\
$\mathbf{2}$ & 24 & Single & Employee & Bachelor & Daughter & 5 years \\
$\mathbf{3}$ & 48 & Married & Housewife & Secondary school & Mother & 2 years \\
$\mathbf{4}$ & 26 & Single & Student & Associate & Son & 2 years and 4 months \\
$\mathbf{5}$ & 42 & Married & Housewife & Diploma & Mother & 8 years \\
$\mathbf{6}$ & 25 & Single & Employee & Master & Sister & 1 year \\
$\mathbf{7}$ & 24 & Single & Student & Bachelor & Daughter & 2 years \\
\hline
\end{tabular}

\section{Data measurement/ collection}

Data were collected in the chemotherapy clinic with prior coordination and with the permission. First, the purpose of the research was explained to them; then, sampling was continued until the data were saturated. The criterion for achieving data saturation was the lack of access to new concepts and codes in subsequent interviews.

Data collection method was semi-structured in-depth interview using open-ended questions, including, "Tell us about your experiences after your breast cancer patient? How has your life changed since your breast cancer patient?" During the interview, the researcher helped participants share their experiences without giving direction. Exploration questions were also used if necessary. The interviews conducted in a quiet place. The duration of each interview was 45-60 minutes, depending on the circumstances and the patience of the participants in one or two sessions. The interviews were typed, verbatim, reviewed, coded, and immediately analyzed by the researcher.

\section{Analyses}

Data analysis was performed directly after with data collection. Data were analyzed using the content analysis approach in a conventional method. In this method, at first, each interview was carefully read to gain an initial understanding, underlined the essential statements and recorded as codes (initial coding). For the initial coding, the words of the participants themselves and the signifying codes (researcher perceptions of the utterances) were used. The codes, which were conceptually similar to each other, were then summarized and regarded as categories and subcategories. The data analysis was performed according to the steps proposed by Graneheim and Lundman [9]. 


\section{Rigor and trustworthiness}

Participants were given the text of coded interviews to confirm their compliance with their experiences, and in some cases, corrections were made. The resulting codes and concepts were consulted and reviewed with experts and collaborators on the research project. Also, several colleagues were asked to stagnate some parts of the text of the interviews for the coding agreement. To confirm the transferability of the findings, patients with different demographic characteristics and experiences in- cluded in the study and the researcher assessed all aspects of behaviors, events and life experiences. The findings confirmability was achieved through a detailed description of the researcher from all stages of the research. Besides, the details of the research were carefully documented to enable the evaluation of external observers.

\section{Results}

Analysis of data led to the extraction of three categories and six subcategories [Table 2].

\begin{tabular}{|c|c|c|}
\hline Main category & Categories & Subcategories \\
\hline \multirow{6}{*}{ Trying to save the patient } & \multirow[t]{2}{*}{ Psychological care while emotional conflict } & Psychological care \\
\hline & & Psychological conflict \\
\hline & \multirow[t]{2}{*}{ Compassionate efforts in painful moments of caring } & Painful moments of care \\
\hline & & Compassionate efforts \\
\hline & \multirow[t]{2}{*}{ Development of capabilities in conflict challenges } & Growth of Capabilities \\
\hline & & Care-treatment challenges \\
\hline
\end{tabular}

\section{Psychological care while emotional conflict}

Participants' experiences showed that they cared for their patients emotionally while they were emotionally conflicted.

\subsection{Psychological care}

The experiences of family members showed that to avoid upsetting and losing patients, they attempted to hide the disease from them and bear the burden of diagnosing the disease alone. Even when the patient was informed of her illness, they took care of her emotional state. A 42-year-old mother underwent a mastectomy expressed her efforts to calm her sick daughter as follows: "The first time my daughter went to the bathroom, she saw herself in the mirror and she doesn't have a breast. She cried a lot in the bathroom, and I could hear her crying. The next day I got rid of all the mirror at home." A 37year-old girl who hidden her mother's illness stated as follows: "Ever since the doctor told me my mother had breast cancer, we still haven't told her. I'm afraid my mother will find out she has cancer."

\subsection{Psychological conflict}

The experiences of family members showed their psychological conflict with the subject of the disease. They were shocked and anxious to find out about the condition and felt anxious, lonely, depressed, restless and uncomfortable. A 24-year-old girl expressed her emotional sensitivity to her mother's mastectomy as: "My mother's illness made me feel a hatred against all the people who were bothering me at that time, I can't erase it from my mind." A 37-year-old daughter also said about the feeling of bitterness in her life following her mother's illness: "Ever since I found out my mother has cancer, life doesn't feel pleasant. Somehow we have not eaten or slept. We all worry about what to do if something happens to my mother."

\section{Compassionate efforts in painful moments of care} The experiences of family members showed that despite the sad moments they experienced during the care, they continued their compassionate efforts to help the patient. 


\subsection{Painful moments of care}

The experiences of the family members of the affected women showed that they had experienced painful moments in caring for the patient, including seeing the side effects of chemotherapy, such as, hair loss and eyebrows, which was very painful for the family. A 48year-old mother of one of the women with breast cancer expressed her unhappiness in the moments of facing the effects of her daughter's chemotherapy as follows: "Ever since my daughter started chemotherapy, she has lost her appearance and her hair has turned yellow and yellow. It is excruciating for a mother to see her daughter in this condition." A 26-year-old sick boy also said about the moment of learning about his mother's illness: "The worst blow of my life came to me when the doctor told me that your mother had breast cancer. It was a tough moment [sighing the sick boy and the sadness on his face], and I was confused. I turned pale and looked at the doctor dumbfounded."

\subsection{Compassionate efforts}

The experiences of the family members of women with breast cancer showed that they made compassionate efforts to improve the patients' mood, attempted to accompany the patient in the situation, and provide extensive support to the patient. A 26-year-old boy from a breast cancer patient stated: "I spent my whole life treating my mother, even because of my mother, I skipped some of my plans to take more care of her." A 42-year-old mother described the efforts for her sick daughter as follows. "His father and I did our best not to miss anything, and we thank God that our efforts are paying off because the doctor told us that the other two stages of chemotherapy would most likely end."

\section{Development of capabilities in conflict chal- lenges}

The experiences of family members showed that facing challenges during patient care has strengthened and developed some capabilities in them.

\subsection{Growth of capabilities}

The experiences of family members of women with breast cancer have shown that the illness of a family member has strengthened and developed some of their abilities because they felt responsible for their patient and intended to care for and protect them. A 26-yearold boy with a breast cancer patient described his sense of duty in caring for his mother as follows: "I was a child when my father died. The burden of our lives was on my mother. If I give my life for my mother, it doesn't compensate any of her hardships." A 24-year-old girl expressed her feelings of growing up and experiencing her mother's illness as follows: "I have come to the point in my life that God says that every problem that comes our way is not a divine and foolish test. I grew up with my mother's illness. I learned a lot, and I grew up, I am afraid of dealing with problems previously. I gained a lot of experience."

\subsection{Care-treatment challenges}

The experiences of family members of women with breast cancer showed that they had difficulties in caring for the patient. They needed capital to pay for treatment, and because they could not afford it, they were forced to borrow. On the other hand, caring for a patient with breast cancer increases work pressure and responsibility among family members and leaves no power to care for other family members. A 42-year-old mother had a mastectomy, said about her daughter's medical expenses: "The cost of treatment was shocking. We spent all our savings. My husband borrowed money from his office, but we still borrow from acquaintances." A 37-year-old girl with breast cancer described her lack of time to care for her family following her mother's illness as follows: "Since this issue [breast cancer] has occurred to my mother, I have less access to my family. I have neglected my children. The children are weak in their education. I used to take care of their lessons and exercises, but now I don't have time." 


\section{Discussion}

Participants' experiences showed that they cared for their patients emotionally while they were emotionally conflicted. Previous studies argue that the hospitalization of one family member causes anxiety, depression and stress in other family members [10], and the pressure of patient care endangers the mental health of caregivers [11]. Caregivers who spend more hours for patients experience more significant stress and lower mental health [12]. In fact, the family is the center of help, relief, and healing. A center that alleviates the psychological pressures on its members and paves the way for their growth and prosperity [13].

The experiences of family members showed that they continued their compassionate efforts to help the patient, despite experiencing painful moments during care and being conflicted with the patient's problems. Diagnosis of cancer is a crisis for the patient and family [14]. Although the family members are responsible for supporting and caring for the patients compassionately, they need support in daily life as well [15].

The experiences of family members showed they facing challenges during patient care has strengthened and developed some capabilities in them. Previous studies argue that the burden of care will impose the family system in negative and positive issues such as exhaustion, getting more support, and adapting to the situation $[16,17]$.

\section{Conclusion}

The experiences of family members of women with breast cancer showed that they had experienced painful moments while caring for the patient. Still, they made a compassionate effort to address the therapeutic care challenges posed by the disease. Despite many psychological conflicts, they attempted to care for their patients. However, they also experienced the growth of some of their capabilities. Therefore, "trying to save the patient" was identified as the essence of the participants' experience. Thus, family members should not be neglected when they care for a chronic patient.

\section{Author contributions}

Conceptualization: ZYB, MR. Formal analysis: MR. Methodology: ZYB, MN, MR. Writing - original draft: ZYB, HRG . Writing - review \& editing: MR.

\section{Conflict of interest}

No declared.

\section{Funding}

None.

\section{Acknowledgments}

This article is the result of a master's thesis in MedicalSurgical Nursing from the School of Nursing, Zabol University of Medical Sciences. The authors appreciate the Zabol University of Medical Sciences and those who participated in the study.

\section{References}

[1] Nekoueifard O, Jahangiri L. Sexual function among patients with breast cancer. J Iran Inst Health Sci Res 2014;13:425-431.

[2] Monfared A, Pakseresht S, Ghanbari A, Atrkar Roshan Z. Health- related quality of life and its related factors among women with breast cancer. Holist Nurs Midwifery J 2013;23:52-62

[3] Samiei siboni F, Anoosheh M, Alhani F, Haji Zadeh. The effect of family counseling on performance scales of quality of life of women with breast cancer undergoing chemotherapy. Adv Nurs Midwifery 2011;20:19-28. https://journals.sbmu.ac.ir/en-jnm/article/view/2312

[4] Safaeian Z, Sadat Hejazi S, Delavar E, Hoseini Azizi T, HaresAbadi M. The relationship between caregiver burden depression, anxiety and stress in family caregivers of cancer patients referred to Imam Reza Hospital in Bojnurd City. Iran J Psychiat Nurs 2017;5:7-13.

[5] Abbasi A, Shamsizadeh M, Asayesh H, Rahmani H, Hosseini S, Talebi M. The relationship between caregiver burden with coping strategies in family caregivers of cancer patients. Iran Nurs Sci Assoc 2013;1:62-71.

[6] Sajadian A, Hidary L, Mokhtari Hesari P. Common breast cancer family care giving problems. Iran J Breast Disease 2015;8:714. 
[7] Heidari Gorji MA, Bouzar Z, Haghshenas M, Kasaeeyan AA, Sadeghi MR, Ardebil MD. Quality of life and depression in caregivers of patients with breast cancer. BMC Res Notes 2012;20;5:310. doi: 10.1186/1756-0500-5-310.

[8] LeSeure P, Chongkham-Ang S. The Experience of Caregivers Living with Cancer Patients: A Systematic Review and MetaSynthesis. J Pers Med 2015;19:406-39. doi: 10.3390/jpm5040406. PMID: 26610573; PMCID: PMC4695863.

[9] Graneheim UH, Lundman B. Qualitative content analysis in nursing research: Content, procedures and measures to achieve trustworthiness. Nurs Educ Today 2004;24:105-123.

[10] Navidian A, Sarhadi M, Keykhaie A, Keykhah R. Psychological reactions of family members of patients hospitalized in critical care and general units compared with general population. Iran J Nurs 2014;26:16-28

[11] Haresabadi M, Bibak B, Hosein Zadeh E, Bayati M, Arki M, Akbari H. Assessing burden of family caregivers of patients with schizophrenia admitted in IMAM REZA hospital Bojnurd 2010. J North Khorasan Uni Med Sci 2012;4:165- 171.

[12] Chung H-Y, Chiou C-J, Chen N-S. Impact of mental health and caregiver burden on family caregivers physical health. Arch Geront Geriatr 2010;50:267-71.
[13] Azizi S, Rahmani A, Ghaderi B. Marital satisfaction in breast cancer patients. J Midwifery Nurs Tabriz 2010;17:50-55

[14] Joulaee A, Joolaee S, Kadivar M, Hajibabaee F. Living with breast cancer: Iranian women's. Int Nurs Rev 2012;59:362-368.

[15] Shorofi SA, Jannati Y, Roohi Moghaddam H. The psychological needs of the families of the patients admitted to intensive care units: A review of literature. Clinic Excellence 2014;3:46-57.

[16] Arshi M, Nazif A, Imani F, Gholamijam F. The relationship between care giving burden and family function of children with cancer. Q J Soci Work 2015;4:35-41.

[17] Rahnama M, Fallahi Khoshkenab M, Seyed Bagher Maddah S, Ahmadi F. Designing a model for spiritual care in rehabilitation of cancer patients. Med Surg Nurs J 2014;3:61-70.

“cite: Younes Barani Z, Rahnama M, Naderifar M, Assadi Bidmeshki E, Rashki Ghalenow M. Family experiences about women with breast cancer: A content analysis study. J Nurs Explor 2021;1 :X . https://doi.org/10.29252/jnexp.1.1.X 